\title{
RAPID TECHNOLOGY ASSESSMENT VIA UNIFIED DEPLOYMENT OF GLOBAL OPTICAL AND VIRTUAL DIAGNOSTICS
}

\author{
Jeffrey D. Jordan, A. Neal Watkins, Gary A. Fleming \& Bradley D. Leighty \\ NASA Langley Research Center \\ Hampton, VA 23681 \\ Richard J. Schwartz, JoAnne L. Ingram, Keith D. Grinstead, Jr. \& Donald M. Oglesby \\ Swales Aerospace, NASA Langley Research Center \\ Hampton, VA 23681 \\ Charles Tyler \\ Air Force Research Laboratory \\ Wright Patterson AFB, OH 45433
}

\begin{abstract}
This paper discusses recent developments in rapid technology assessment resulting from an active collaboration between researchers at the Air Force Research Laboratory (AFRL) at Wright Patterson Air Force Base (WPAFB) and the NASA Langley Research Center (LaRC). This program targets the unified development and deployment of global measurement technologies coupled with a virtual diagnostic interface to enable the comparative evaluation of experimental and computational results. Continuing efforts focus on the development of seamless data translation methods to enable integration of data sets of disparate file format in a common platform. Results from a successful lowspeed wind tunnel test at WPAFB in which global surface pressure distributions were acquired simultaneously with model deformation and geometry measurements are discussed and comparatively evaluated with numerical simulations. Intensity- and lifetime-based pressure-sensitive paint (PSP) and projection moiré interferometry (PMI) results are presented within the context of rapid technology assessment to enable simulation-based R\&D.
\end{abstract}

\section{$\underline{\text { INTRODUCTION }}$}

Simulation-based research and development (SBR\&D) strategies have long been pursued to enable the prediction of vehicle and component technology performance and its subsequent impact to the vehicle mission. Realizing these goals requires the development of rapid technology assessment tools to source the information necessary for code optimization and validation quickly and comprehensively, mitigating significantly the length and cost of the technology assessment process and comprehensive vehicle design. Conventional vehicle design cycles require the fabrication of two sub-scale test models. The first model is typically constructed early in the design cycle and predominantly un-instrumented, relying on facility balance force and moment data to provide a measure of performance over the vehicle flight envelope. A second model is subsequently constructed that is highly instrumented with surface pressure measurement systems necessary for aerodynamic loads calculations. The fabrication of this so-called "loads" model is a significant contributor to the cost and time required to acquire performance data for the optimization and validation of numerical methods codes, such as computational fluid dynamics (CFD) and finite element analysis (FEA). Further, the SBR\&D mission encompasses additional objectives that focus on the establishment of a centralized and unified data base that enables impact prediction of virtually any technology component imaginable.

As a result, there remains a critical need for the development of unified global diagnostic technologies that can be deployed simultaneously to acquire aerodynamic cause-and-effect relationships. ${ }^{1}$ Moreover, a common interface is required that enables the comparative evaluation of experimental and computational results, seamlessly translating data files of disparate formats across the platform. Such a capability would provide the aerodynamic designer with a comprehensive insight of the technology assessment process, and in doing so provide mission simulation analyses to rapidly assess the impact of the design or component technology on the vehicle mission. This perspective provides useful knowledge to mission planners for developing near- and long-term investment strategies that target the highest level objectives of the organization. For example, the vision of revolutionary aerospace vehicles encompasses a number of advanced technologies based on morphing and adaptive structures that are comprised of lightweight, multi-functional and highly deformable materials. Flight qualification of experimental structural and control surface materials and technologies will require wind tunnel testing on subscale models, the characterization of which will require advanced diagnostic technologies capable of measuring simultaneously the model 3D geometry/deformation and the global surface pressure distribution. In addition, a 
virtual interface is required to enable the comparative evaluation of experimental and computational data to support the rapid assessment of revolutionary aerospace vehicle technologies.

This paper focuses on an active collaboration ${ }^{2}$ between researchers at the NASA LaRC and the AFRL at WPAFB and their contractors. Developmental efforts toward advancing the state-of-the-art in time-resolved PSP and PMI measurement techniques to recover surface pressure and model deformation and geometry information, respectively, are highlighted. A newly developed technique called projection/resection of optical dots (PROD) was deployed that enables the nearreal time visualization of surface pressure distributions on 3D model geometries. In addition, a software system called the virtual diagnostics interface (ViDI), consisting of an interactive, 3D environment of the SARL facility, has been developed to enable both the pre-test optimization and planning of the instrumentation deployment, and the comparative evaluation of global surface pressure distributions (PSP) and model deformation (PMI). The ViDI platform is being further developed as the hub by which data streams of disparate file formats are comparatively evaluated using seamless data translation (SDT) techniques. This paper discusses the results from a test of a lambda wing configuration model in the Subsonic Aerodynamic Research Laboratory (SARL) at WPAFB, a 7 x 10-foot low-speed wind tunnel facility. A discussion of the individual diagnostics deployed in this unified test (ViDI, PROD, PSP and PMI) is followed by a review of selected test results and future directions.

\section{Virtual Diagnostics Interface (ViDI)}

The virtual diagnostics interface (ViDI) is a software ensemble that provides unified data handling and interactive $3 \mathrm{D}$ displays for pretest planning, real time data visualization and post test analysis to enable: 1) test planning and optimization remotely with no facility impact, 2) comparative evaluation of experimental and computational data sets of disparate file formats (SDT) for code optimization and validation, and the 3) establishment of a central hub to source, store and retrieve experimental results and display them in nearreal time to enable the characterization of the impact of technologies on the vehicle aerodynamics, structures, controls and integration components to achieve a true rapid technology assessment capability. And in doing so, manifest as an integral component in the SBR\&D fundamental sciences foundation that feeds the mission simulation and campaign elements as illustrated in Figure 1.

ViDI itself has evolved from a decade long application of three-dimensional computer modeling for pre-test planning and post test data visualization. ${ }^{3}$ In this approach, state-of-the-art commercial-off-the-shelf
(COTS) software for the Windows environment enables the researcher to merge accurate three-dimensional wind tunnel geometries with the CAD files developed to build the actual wind tunnel test models.

Creating a virtual representation of the model and test section elements provides a platform to design the instrumentation layout. All hardware elements can be easily manipulated within the virtual environment to provide a mechanism to reconcile facility issues such as optical access and test section real estate to secure hardware. This capability is particularly advantageous when multiple instrumentation systems are to be deployed or installed simultaneously. The ViDI environment enables researchers to simulate multiple test configurations to ensure measurement system efficacy in all regions of interest in the test matrix under varying parameters such as angles of attack or flap configurations. Using the pre-test planning aspect of ViDI, these concerns can be met at the desktop, prior to the start of a wind tunnel test, and eliminate the high cost of reconfiguring hardware in the facility. ViDI has taken this capability and expanded upon it.

For the test in the SARL at WPAFB, ViDI-based simulations helped to clarify the optimal architecture for the planar Doppler velocimetry (PDV) laser light sheet with respect to the model and the wind tunnel. The program simulation translated the laser sheet along a specified length of the test section to create a 30 frameper-second animation of the movement. Locations in which the laser light sheet was blocked by window frames were clearly depicted, as shown in Figure 2, with angles-of-attack sweeps revealing regions of the airframe that could be investigated with a given hardware arrangement. Through these simulations, the ViDI system reduced significantly the time and cost associated with optimizing the multi-component PDV instrumentation.

Similarly, the ViDI system also maintains the capability to evaluate optical perspective and distortion effects within the virtual wind tunnel environment to predetermine optimal optics and lens configurations for camera systems integral to advance global diagnostics. In this approach, the field of view angle for each camera is determined and coupled with the known aspect ratio and resolution of each camera. In doing so, the rendered virtual image is a realistic facsimile of what the actual camera will encounter in the wind tunnel facility. Simulated images can be further exploited to visualize the impact of noise sources (e.g., vibrational blur, stray light) on the recovered data, providing a mechanism to design and evaluate strategies for optimizing data fidelity over varying environmental conditions.

A second aspect of ViDI was implemented in the SARL test at WPAFB to allow the mapping of surface pressure data onto a computer-generated model in "near-real 
time". This capability currently exploits texture mapping techniques to place an image of the data onto a surface, allowing the user to examine the data in a threedimensional virtual environment. PMI-based measurements provide the true aero-loaded model geometry on which PSP-based surface pressure distributions are mapped, and it is the objective of this effort to develop rapid data analysis methods for both PSP and PMI systems. To augment this capability, an alternative approach to providing near-real time threedimensional visualization of surface pressure distributions on 3D model geometries is also under development. This capability relies on a technique called projection/resection of optical dots (PROD). PROD is a straightforward method that directly suits the needs of the COTS three-dimensional modeling software used to perform the resection of planar image data back onto the real three dimensional surface.

The PROD technique requires the acquisition of an image of a grid of equi-spaced dots on the surface under investigation. This is achieved using a computer projector coupled with a program that generates a field of dots of any desired size, color, spacing and rotational orientation. This dot pattern is projected onto the model and an image is captured with the camera used for the PSP measurements. Two additional images are then acquired with all of the dots set to the same color as the dot background, followed by an image of the illuminated PSP surface. Because the emission from the PSP-coated model is so much brighter than the surrounding background, this image can be inverted and used as a mask to limit the region of interest to the painted portions of the model.

A custom program was developed to locate the dot centroids and sort them for use in a bilinear image warping routine, where the user selects the first dot in each row and the program determines the location of the remaining dots automatically. Once all of the centroids are located and sorted into the correct rows and columns, the program maps the locations of the centroids to a grid that is perfectly square. This has the effect of "dewarping" the image; thereby, providing the image transform necessary to re-warp the data onto the curved surface of the computer model, recreating the proper data patterns on the 3D surface geometry.

In this approach, PROD images are acquired and processed just prior to the test run to recover the dewarped mapping coordinates. The processed PSP data is subsequently de-warped and applied to the computer geometry. This texture-mapped three-dimensional computer model can then be viewed and analyzed immediately. An interface is under development that allows the user to place the mouse on the surface and determine both the magnitude of the deflection and the surface pressure at any point or region of interest on the model. Similarly, the off-body velocity data can also be imported into the data visualization environment, providing greater insight into aerodynamic cause and effect relationships.

In the future, ViDI will encompass a range of user needs and technology levels. These components are complimentary, starting from a very basic educational guide of a facility through high-end productivity tools for real time data processing and analysis. Moreover, these elements are vital to support global collaboration throughout the vehicle design cycle and enable contributions from non-local individuals in a near-real time basis. This objective is being pursued through the implementation of seamless data translation mechanisms coupled with modern data portability strategies.

\section{$\underline{\text { Seamless Data Translation }}$}

In order for the ViDI components to provide maximum utility they must be linked together by an infrastructure that allows them to interact seamlessly. By establishing a set of open standards based on commercial state of the art information technology, ViDI becomes a backbone to which an unlimited number of additional elements can be connected, providing a powerful platform for information processing.

Paramount to unifying experimental and computational results within the ViDI environment is the establishment of a common file format that supports a self-descriptive header. A file format with a self-descriptive header refers to a type of file that can contain almost any type of data. Information about the type of data and how to use it is stored in the header portion of the file. A modern trend in file design has been to allow several different types of information (for example a 3D geometry as well as images) within one file. Information within the file would be organized under a directory, or folder structure, analogous to a computer hard drive. In this concept, ViDI would specify a header structure that would allow one or more types of data (e.g., PSP images, calibration data and processing values) to be stored in one file. System flexibility will allow data from multiple instruments to be stored in the same file. By laying out a common set of logical rules that can allow data files to be automatically expanded and contracted as needed by individual applications, various types of information can be handled in a fast and efficient manor, eliminating the need for the user to translate data manually. The HDF5 file format, developed by the National Center for Super Computing Applications (NCSA) ${ }^{4}$ specifically to handle very large data sets, is being pursued toward this end.

A new standard for data manipulation over networks (and the Internet) is emerging at a rapid pace. This is called Web Services, ${ }^{5}$ and is currently comprised of a number of different technologies that either complement or compete with one another. Web Services will allow 
more efficient ways of handling large sets of data between large numbers of computers and facilitate distributed computing. The heart of this technology is based on XML (extensible markup language), ${ }^{6}$ which is both a file format and programming language. Leading software firms currently employ XML as the backbone for advanced network operations. Databases, office applications, and other common programming packages over a wide variety of platforms are currently using XML. However, XML is based purely on ASCII text, and not binary data. This excludes its applicability to handle large data sets intrinsically. One compromise would exploit the XML format as a wrapper to the HDF file. The XML header will contain basic information about the HDF file, which will be used for cataloging and routing the file over the network, with the actual data held in HDF format.

A common data base of three-dimensional geometries will be available to the end user. Wind tunnels will be modeled in major components, allowing the end user to specify a computer model that includes the entire facility, or just one portion, such as the test section. This database would be created in the Non-Uniform Rational B Spline (NURBS)-based Initial Graphic Exchange Specification (IGES) 3D computer model file format, as IGES is one of the most widely used formats among multiple platforms. Thus, an IGES file can feed the visualization aspects of ViDI, a mesh generation module for CFD or FEA, or a CAD workstation for detail enhancement or modification. The IGES file would then be "wrapped" in an HDF5 file for ease of incorporating it with the comprehensive test data set. In addition to the wind tunnel, each major sting component will be included in the library, with information embedded into the three dimensional model to specify the type of connections (physical and electrical) that can be made at each end. Other parameters, such as mass, modulus of elasticity, and even the last user and location where the hardware was stored can be incorporated as well.

The future goals that have to be met in order to keep wind tunnel testing affordable are numerous and complex. The virtual diagnostic interface will play a key role in integrating a variety of advanced diagnostic capabilities with wind tunnel facilities, computational resources, and even flight data to enable rapid technology assessment capabilities critical to meeting the objectives of current and future advanced aerospace systems.

\section{$\underline{\text { Pressure Sensitive Paint (PSP) }}$}

Pressure sensitive paint (PSP) is an optical diagnostic capable of recovering global surface pressure distributions on test models. $1,7-17,25,26$ The technique exploits oxygen-sensitive luminescent molecules that are dispersed in polymer binders, or paints. In wind tunnel applications, the PSP is applied to the model by conventional paint spraying techniques. Light sources such as blue LED arrays are mounted external to the test section to illuminate the painted model and effect luminescence emission from the entrapped oxygensensitive molecules. For the majority of pressure paints, PSP emission occurs in the orange region of the visible spectrum $(\sim 650 \mathrm{~nm})$, the intensity of which is inversely proportional to pressure such that brighter regions in the paint emission indicate lower pressure relative to the darker areas. Scientific-grade CCD cameras with spectral band-pass filters to discriminate between the excitation (blue) and emission (orange) signals, capture the intensity image of the PSP-coated model surface, providing a means to recover global surface pressure distributions on test articles of interest.

PSP measurement systems all employ a ratio of image pairs to compensate for intensity non-uniformity due to sources other than pressure, the most significant of which are paint application and illumination heterogeneity. In the conventional approach, PSP images acquired either prior to or immediately following tunnel operation (wind off) are ratioed with images acquired at each tunnel condition (wind on). This "intensity method" requires the acquisition of associated wind off images at each model position, attitude and configuration to allow ratioing with the corresponding wind-on image. In addition to the need to bring the tunnel down or to an idle condition for extended periods, this approach also exasperates the temperature sensitivity inherent to PSP systems. The latter point results from the significant differences in tunnel static temperature between wind-on and wind-off conditions, which occurs both in open-draft and closed-loop wind tunnel circuits. Like all good sensors, PSP systems are temperature sensitive. In typical operation, PSP temperature sensitivity is compensated for using image software that forces the PSP intensity-ratio image data to agree with on-model pressure tap data. This in situ calibration technique, requiring only a modest population of pressure taps $(\sim 6)$, has formed the foundation by which production PSP diagnostic systems have achieved quantitative and traceable results.

A major element of the current AFRL-NASA Langley collaboration $^{2}$ focuses on the development of PSP measurement techniques that are insensitive to temperature; thereby, eliminating the need for on-model instrumentation. Achieving this goal would significantly expedite the technology assessment process by enabling the recovery of aerodynamic loads on completely uninstrumented models created by rapid prototyping techniques. Toward this end, we are developing a timeresolved PSP measurement system ${ }^{12-17}$ that does not require the acquisition of wind-off images. Rather, an interline-transfer CCD camera coupled with pulsed illumination from LED light sources provides a mechanism to recover images of the PSP intensity decay at two discrete times following the LED excitation pulse. 
This temporal resolution allows the acquisition of both reference (short delay following the excitation pulse) and run images (longer delay with wider temporal gate) at condition, eliminating the need for wind-off image data. Moreover, in time-resolved, or "lifetime" mode, the PSP run and reference images are both acquired with the model under load; thereby, reducing or eliminating the need to apply image photogrammetry techniques to warp the spatially deformed wind-on image to the unloaded wind-off image prior to ratioing. Finally, acquiring both images at condition with the same model surface temperature reduces PSP error due to temperature sensitivity, minimizing or eliminating the need for pressure-tap data. During the April 2003 RTA SARL entry, the Langley Team successfully deployed both intensity- and lifetime-based PSP measurement systems simultaneously with a PMI system that recovered model deformation and 3D model geometry information. These data sets were combined using ViDI to apply the recovered surface pressure distributions (PSP) to the aero-loaded and deformed model 3D geometry (PMI), and simultaneously compared to the CFD solution for the same condition.

\section{$\underline{\text { Projection Moiré Interferometry (PMI) }}$}

PMI is a video-based, non-contacting measurement technique capable of obtaining spatially continuous measurements of out-of-plane structural deformations. The technique is well suited to measuring the deformation of aeroelastic vehicle components and aerodynamic control surfaces. ${ }^{1,}{ }^{18-25}$ The deformation measurements can be used to refine CFD grid geometries of the model under test to reflect the actual, aero-loaded model geometry. The deformation data can also be used to improve the data quality of other instruments used during the test.

PMI as applied to measuring wind tunnel model deformation is shown schematically in Figure 3a. A projection system is used to project a grid of equispaced, parallel lines onto the wind tunnel model surface. A CCD camera positioned to view the model obliquely is used to record images of the grid pattern projected onto the surface (Figure $3 b$ ). Changes in the wing shape, either by aerodynamic loading or control surface actuation, will cause the projected grid lines to lie in different spatial locations on the model surface. Images of the projected grid are acquired while the model is subjected to different states of stress or actuation. These images are computationally interfered with a reference grid to produce interferograms as shown in Figure 3c. The interferograms contain moiré fringes, which are contours of the out-of-plane object deformation. The interferograms are then further processed to reconstruct the spatially continuous object shape or deformation (Figure 3d). The PMI system measurement accuracy and resolution are primarily dependent upon the projected grid pitch, video camera field-of-view, optical modulation transfer function, and illumination/observation angles. ${ }^{18}$ However, as a conservative rule-of-thumb, the out-of-plane deformation measurement accuracy and resolution of a PMI system can be approximated at 1/1500 and 1/2500 of the field-of-view, respectively.

\section{EXPERIMENTAL}

The lambda wing configuration model tested was constructed from resin materials using stereolithography techniques. The model was equipped with 64 pressure taps on the upper surface, six of which were used to apply an in situ calibration for the PSP data. The model was first coated with a screen layer (ISSI proprietary formulation $)^{26}$ to mask the inherent fluorescence of the model material and to provide a uniform surface on which to apply the PSP. A single-coat FIB-based ${ }^{26}$ PSP was applied over this and used to recover both intensityand lifetime-based PSP image data. The matte finish of the paint is ideally suited for the PMI diagnostic, which images the reflection of the IR laser source from the deforming model surface.

The PSP instrumentation consisted of a scientific-grade, back-lit, 512 x 512 pixel CCD camera digitized at 16-bit $\mathrm{A} / \mathrm{D}$ resolution (Pixelvision, Spectravideo) for the intensity-based measurements, an interline-transfer/ hardware accumulator, $1 \mathrm{~K} \times 1 \mathrm{~K}$ pixel CCD (12-bit A/D) for the lifetime measurements and four, 10-cm-diameter LED arrays $(405 \mathrm{~nm}){ }^{26}$ The LED illumination sources were operated in long-pulse and square-wave modulated $(5 \mathrm{kHz})$ modes for the intensity- and lifetime-based measurements, respectively. For the intensity-based measurements, 16 images were acquired with integration times on the order of one second and averaged prior to the application of the in situ calibration to the surface pressure tap data.

The PMI system hardware consisted of a 1392 x 1040 pixel CCD camera, and a custom-fabricated projector head for grid line projection. The projector illumination source was a fiber-optically coupled laser diode emitting at $795 \mathrm{~nm}$. The laser line width was nominally $3 \mathrm{~nm}$ full-width at half-maximum (FWHM), which minimized the appearance of speckle on the diffuse scattering surface created by the PSP. The laser light was fiberoptically coupled into the rear of the projector head, and was passed through a Ronchi ruling for generation of the grid lines. The grid lines were then projected onto the model surface using a standard single-lens-reflex (SLR) camera lens. For each test condition, 100 images of the projected grid pattern were acquired at $30 \mathrm{~Hz}$ and digitized using the CCD camera and stored locally on a PC-compatible computer. Each image of the 100-image sets was subsequently processed and analyzed to determine the average and rms model deformation for each test condition. 
PROD measurements were performed a single time prior to wind tunnel operation by acquiring images with the PSP camera of a dot pattern projected onto the model at the two angles of attack under investigation (12, 20 degrees). A standard presentation-type LCD projector was used in concert with a laptop PC running a code that generates a tunable dot pattern (e.g., size, color, density, orientation, background). These images were processed according to procedures outlined in the next section to recover the transformation coordinates necessary to map the 2D PSP pressure distributions to the true 3D (but not aero-loaded) model geometry for visualization and analysis purposes.

\section{$\underline{\text { RESULTS AND DISCUSSION }}$}

Global intensity- and lifetime-based PSP measurements were performed in a unified manner with PMI and PROD in the SARL in April 2003 on a lambda wing configuration model (Figure 4). Installation of the measurement system hardware and cabling and PMI system/facility calibration was achieved in approximately one shift. This was accomplished by exploiting the pre-test planning capabilities of ViDI, enabling instrumentation layout design to occur from a desktop PC at NASA Langley, nearly $1000 \mathrm{~km}$ away from WPAFB. Figure 4 (right image) shows the CCD cameras and illumination sources (PSP LED arrays, PMI fiber-optic-coupled laser projection system) mounted above the SARL test section. A keyboard-video-mouse (KVM) extender system (inset, upper left corner) was incorporated to enable remote operation of the data acquisition PCs (locating in the control room $\sim 100 \mathrm{~m}$ distance) from the top of the test section.

Multiple configurations of the lambda wing model were tested with varying inboard, mid-board and outboard aileron angles $(-20,0,+20$ degrees $)$ at two model angles of attack (12 and 20 degrees) at Mach numbers 0.2 and 0.4. Typical data acquisition sequences for a given configuration consisted of pre-run wind off reference data acquisition, followed by a pitch/pause (12, 20 degrees) at condition $(\mathrm{M}=0.2)$, with a reverse pitch/pause at $\mathrm{M}=0.4$ Reference data was also acquired during post-run idle operation of the tunnel. A single set of PROD images were acquired on the base configuration during the pre-test setup to enable the near-real time visualization of the surface pressure distribution mapped to the 3D model geometry.

Recovered two-dimensional PSP images were analyzed using the Greenboot ${ }^{11}$ software running on a Linuxbased PC. A second software package developed at NASA Ames Research Center by Edward Schairer, called Legato, was also employed during testing to enable rapid data analysis on a Windows-based PC. Multiple PSP images (e.g., 16 frames. 1-sec integration) acquired at condition and at tunnel idle were automatically background subtracted and averaged using the data acquisition code written at Langley using LabView. Photogrammetry techniques were used to align and ratio the averaged images prior to applying an in situ calibration ${ }^{1,7-17,25}$ of the PSP data to surface pressure taps to compensate for PSP temperature sensitivity and other intensity artifacts not related to surface pressure changes. Typical results are shown in Figure 5 for the base configuration at the four conditions investigated. The data clearly show the effect of Mach number and model angle of attack on the recovered surface pressure distributions. Even under these lowspeed conditions, the low-pressure regions associated with model-tip and leading-edge vortices are evident, with the global spatial resolution elucidating the vortex interaction that occurs along the wing and main body junction. In a manual fashion, these data sets were produced in near-real time during the SARL test entry; thereby, providing a mechanism to further investigate spurious or unpredicted behavior, while ensuring the fidelity of the recovered PSP data sets.

Lifetime PSP images were also acquired as described above using pulsed illumination from the LED arrays and the on-chip integration capabilities of the IT-CCD camera. A two-gate lifetime method ${ }^{1,12-17}$ was applied (G1: $3-8 \mu \mathrm{s} ; \mathrm{G} 2: 15-100 \mu \mathrm{s})$ using a 5 - $\mu \mathrm{s}$ LED pulse width operating at a frequency of $5 \mathrm{kHz}$. In this strategy, PSP images were acquired by integrating photons during the specified G1 and G2 gate widths over several thousand pulses from the LED array. At $5 \mathrm{kHz}$ excitation frequency, one-second integration times were typically required to achieve near saturation of the CCD pixel full-well capacity (60,000 electrons digitized at 12bit resolution). Sixteen images were acquired for each temporal gate and averaged prior to directly ratioing the G1/G2 average images followed by the application of an in situ calibration using surface pressure tap data. Because only four, $10-\mathrm{cm}$-diameter LED arrays were used, the lifetime experiment was excitation-light limited. To compensate for this, the spatial resolution of the lifetime images was compromised by a four-by-four binning process (16 adjacent pixels collapsed to one) to improve the measurement system signal-to-noise ratio. No additional correction of the data was invoked. A typical lifetime-based pressure distribution is shown in Figure 6, and compared to an intensity-based PSP result for the same condition. To enable this comparison, the pressure scales were non-dimensionalized (pressure coefficient, $\mathrm{Cp}$ ) to account for differences in barometric pressure on the two different run days under which the intensity and lifetime PSP experiments were performed. The data reveal good agreement between the intensityand lifetime-based results. Differences in the amplitude of the low-pressure region on the wing leading edge are attributed to the sedimentation of tunnel debris (e.g., grease, oil) in the elliptical region evident in the lifetime image. This caused a reduction in the PSP sensitivity, manifesting in the apparently higher pressure associated with the vortex shed in that region. That 
notwithstanding, these results show promise for the unified deployment of lifetime PSP measurement systems in rapid tech assessment strategies for recovering surface pressure distributions without the need for acquiring wind-off (or idle) reference data.

A global uncertainty analysis was performed on all recovered PSP image data with results showing rms pressure error (relative to pressure taps) in the range of $0.2 \pm 0.1$ and $2 \pm 0.4 \mathrm{kPa}$, for the intensity- and lifetimebased results, respectively.

PMI-based model deformation results for the base configuration are shown in Figure 7. In general, wingtip deflection was on the order of $3 \mathrm{~mm}$ at $\mathrm{M}=0.2$, and ranged from $7-10 \mathrm{~mm}$ at $\mathrm{M}=0.4$. Figure 7 shows a 2D wing-bending profile at $25 \%$ chord. These data reveal a relatively complex bending profile, with the inboard portions of the model bending down with a total displacement inboard-to-outboard of $10 \mathrm{~mm}$ nominally. Under these experimental conditions, the standard deviation of the wing bending was calculated to be \pm 1.0 $\mathrm{mm}$ at the wing tip, with PMI system random noise $( \pm$ $1 \sigma)$ of $\pm 0.15 \mathrm{~mm}$.

To further exploit these unified data sets, the ViDI system was employed to resect both the PMI and PSP data into a common visualization. This was done with the same three-dimensional geometry files, visualization and design tools used to plan the experiment prior to the wind tunnel entry. Both the application of PMI deformation data and the PSP color contours were applied directly from bitmap-based data, albeit through two different procedures as illustrated in Figure 8. The PMI deformation data was applied to the original, nondeformed geometry using a technique called displacement mapping. Displacement mapping, like the texture mapping used for PSP, requires that the imagery contained in a bitmap file be carefully aligned with the geometry. It also requires that optical and perspective distortions be eliminated from the data set. During standard PMI data processing, the data undergoes a similar de-warping procedure as the PROD technique, resulting in an image that appears to be taken from directly above the test subject. Thus, the data can be directly aligned on the geometry by matching recognizable aspects of the image (leading and trailing edges, wing root juncture or dedicated fiduciary marks) with the corresponding features in the virtual environment. Once aligned with the geometry, the computer program will read in the intensity of each pixel in the PMI data bitmap (an 8-bit gray scale image). The geometry located below any given pixel with a non-zero value will be displaced normal to the plane of projection upon which the bit map is set, or false-colored as shown in Figure 9. Thus, for a bitmap aligned with the top view, the geometry displacement would be directly straight up (or down, depending upon offset sign), which is precisely what the PMI instrument has measured. The magnitude of the deflection is adjustable, so a 1:1 scale displacement can be displayed, or the magnitude of the displacement can be scaled to exaggerate the displacement to aid the visualization process. This methodology relies on the region of geometry being deformed containing a mesh with sufficient resolution to display the surface contours adequately. The resolution will vary based on the nature of the displacement, with a simple bend and twist requiring significantly less resolution than a complex sinusoidal variation.

PSP data mapping or resection is described in detail in the previous section of this paper discussing PROD. Like PMI, a de-warped image that removes the optical and perspective distortion is required. PROD or other de-warping technologies can be used for this aspect of the image processing. Again, like the PMI data, the PSP data requires that the bitmap be aligned with the model geometry. For PSP, however, a color bitmap (from 8 to 24 bits per pixel) is used, and applied as a "texture map", or decal, to the surface as shown in the companion image in Figure 9. To demonstrate the efficacy of the ViDI system for comparatively evaluating experimental (EFD) and computational (CFD) results, a CFD solution was mapped to the opposite wing of the lambda wing base configuration geometry as the recovered surface pressure distribution (PSP). The Figure 9 PSP/CFD comparison illustrates simultaneously the need for seamless data translation tools to reconcile data palette and scale disparities, and the value of a 3D visualization to quickly identify unpredicted behavior and to guide the next course of action (i.e., so-called "useful knowledge") in near-real time. In the near future, this strategy will provide mechanisms that span the gamut of possibilities that include simply altering the test matrix to better understanding the flow physics, improving the fidelity of the numerical simulation code, deforming/altering the model grid geometry to generate a new model using rapid model prototyping techniques for a subsequent rapid technology assessment, as well as providing the new geometry in a common format to enable optimization of predictive methods.

\section{$\underline{\text { SUMMARY }}$}

Combined, these results demonstrate the efficacy of this rapid technology assessment strategy for providing nearreal time comparative evaluation of disparate experimental and computational results. Continuing efforts focus on increasing the level of automation of both the PSP and PMI data acquisition and analysis systems. A three-gate lifetime PSP system, capable of recovering simultaneous pressure and temperature distributions, is under development and data sets acquired during the SARL 2003 entry are contributing to its optimization. Seamless data translation schemes based on HDF are being integrated to facilitate the nearreal time comparative evaluation of experimental and computational results. Additional activities endeavor to 
cooperatively develop software capabilities to enable grid deformation based on PMI-recovered model geometry data, and exploit this more accurate representation of the loaded vehicle to optimize computational models and form the platform on which all experimental results are analyzed, displayed and comparatively evaluated with predicted results. Combined, these efforts will produce the tools essential to rapid technology assessment and its contributions to simulation-based R\&D.

\section{$\underline{\text { ACKNOWLEDGEMENTS }}$}

This collaborative effort has been made possible through generous support from the Air Vehicles Directorate of the AFRL at WPAFB operating under sanction of NASA Langley Research Center Interagency Agreement IA1-568, Annex 4. The NASA Langley Rapid Tech Assessment Team gratefully acknowledges the critical efforts of AFRL personnel Raymond Raber and Rick Gillium that enabled this research testing effort in SARL. We also thank Edward Schairer of the NASA Ames Research Center for making the Legato code available for our use. We also acknowledge the vision of Thomas Tighe and the advocacy of Gary Dale at AFRL/WPAFB for fostering this collaborative effort.

\section{REFERENCES}

1.) Jordan, J., Watkins, A., Fleming, G., South, B., Schwartz, R., Oglesby, D., Ingram, J., and Tominack, B., 2003, "Development of Rapid Technology Assessment Tools-A New Paradigm in Aerodynamic Design, Proceedings of the $49^{\text {th }}$ International Instrumentation Symposium.

2.) AFRL NASA Langley collaborative effort sanctioned under NASA Interagency Agreement IA1-568, Annex 4, 2002, "Advanced Optical Measurement Diagnostics for SimulationBased R\&D."

3.) Schwartz, R., 1995, "Virtual Facilities: Imaging the Flow," Aerospace America, July, pp. 23 - 26

4.) The National Center for Supercomputing Applications home page for HDF: http://hdf.ncsa.uiuc.edu/.

5.) Newcomber, E., Understanding Web Services, $X M L$, WSDL, SOAP and UDDI, Addison-Wesley, 2002. ISBN 0201-75081-3.

6.) Dalvi, D., et al., Professional XML for .Net Developers, Wrox Press Ltd., 2001. ISBN 1-861005-31-8.

7.) Crites, R., 1993, "Measurement Techniques- Pressure Sensitive Paint Technique," Lecture Series 1993-05, von Karman Institute for Fluid Dynamics.

8.) McLachlan, B., and Bell, J., 1995, "Pressure-Sensitive Paint in Aerodynamic Testing," Experimental Thermal and Fluid Science, Vol. 10, pp. 470-485.

9.) McLachlan, B., Kavandi, J., Callis, J., Gouterman, M., Green, E., and Khalil, G., 1993, "Surface Pressure Field Mapping Using Luminescent Coatings," Experiments in Fluids, Vol. 14, pp. 33-41.

10.) Kavandi, J., Callis, J., Gouterman, M., Khalil, G., Wright, D., Green, E., Burns, D., and McLachlan, B., 1990, "Luminescent Barometry in Wind Tunnels," Rev. Sci. Instrum., Vol. 61, pp. 3340-3347.
11.) Bell, J., and McLachlan, B., "Image Registration for Pressure-Sensitive Paint Applications," Experiments in Fluids, Vol. 22, pp. 78-86

12.) Mitsuo, K., Egami, Y., Asai, K., Suzuki, H., and Mizushima, H., 2002, "Development of Lifetime Imaging System for Pressure-Sensitive Paint," AIAA-2002-2909.

13.) Goss, L., 2001, "Implementation of Lifetime-Based PSP Techniques in Large Scale Wind Tunnels," Proceedings of the $8^{\text {th }}$ Pressure Sensitive Paint Workshop, Hampton, VA.

14.) Bell, J., 2002, "Lifetime-Based Pressure Sensitive Paint Measurements," Proceedings of the $9^{\text {th }}$ Pressure Sensitive Paint Workshop, Washington, DC.

15.) Engler, R., and Klein, C., 1997, "DLR PSP System: Intensity and Lifetime Measurements," ICIASF '97 Record, International Congress on Instrumentation in Aerospace Simulation Facilities, Pacific Grove, CA, pp. 46-56.

16.) Holmes, J., 1998, "Analysis of Radiometric, Lifetime and Fluorescent Lifetime Imaging for Pressure Sensitive Paint," Aeronautical Journal, 0001-9240, Vol. 102, pp. 189194.

17.) Drouillard II, F., Fisher, C., Linne, M., Middleton, N., Fiechtner, G., Goss, L., and Gord, J., "Evaluation of New Camera Architectures for Pressure Sensitive Paint Measurements," AIAA-2000-2524.

18.) Patorski, K., 1993, Handbook of the Moiré Fringe Techniques, Elsevier Science Publishers, pp. vii - xi, pp. 372373.

19.) Waszak, M., Jenkins, L., and Ifju, P., 2201, "Stability and Control Properties of an Aeroelastic Fixed Wing Micro Aerial Vehicle", AIAA-2001-4005.

20.) Fleming, G., and Gorton, S., 2000, "Measurement of Rotorcraft Blade Deformation Using Projection Moiré Interferometry," J. of Shock and Vibration, Vol. 7.

21.) Fleming, G., Soto, H., and South, B., 2002, "Projection Moiré Interferometry for Rotorcraft Applications: Measurements of Active Twist Rotor Blades," Proceedings of the American Helicopter Society $58^{\text {th }}$ Annual Forum, Montreal, Canada.

22.) Fleming, G., Bartram, S., Waszak, M., and Jenkins, L., 2001, "Projection Moiré Interferometry Measurements of Micro Air Vehicle Wings," Proceedings of SPIE, Vol. 4448, pp. $90-101$.

23.) Fleming, G., and Burner, A., 1999, "Deformation Measurements of Smart Aerodynamic Surfaces," Proceedings of SPIE, Vol. 3783, pp. $228-238$.

24.) Florance, J., et al., 2003, "Contributions of the NASA Langley Research Center to the DARPA/AFRL/NASA/Northrop Grumman Smart Wing Program," AIAA-2003-1961.

25.) Fleming, G., et al., 2002, "Unified Instrumentation: Examining the Simultaneous Application of Advanced Measurement Techniques for Increased Wind Tunnel Testing Capability," AIAA-2002-3244.

26.) Pressure paints and PSP hardware, software and services are available commercially from Innovative Scientific Solutions, Inc. (ISSI), Dayton, OH, www.innssi.com. 


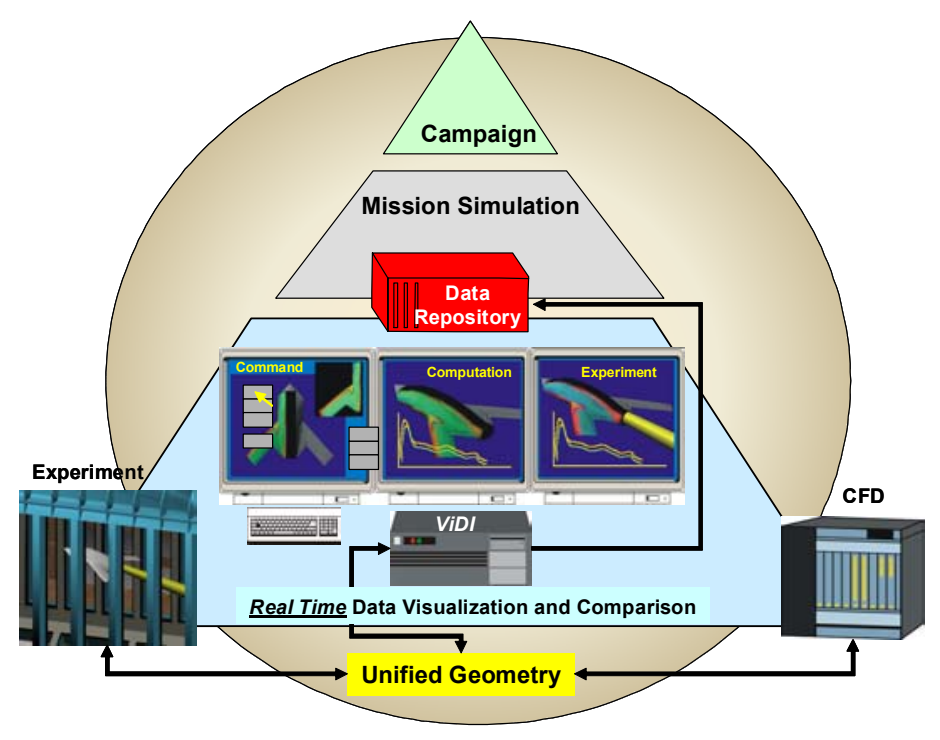

Figure 1. AFRL simulation-based research and development (SBR\&D) pyramid and the integrated role of the virtual diagnostics interface (ViDI) with experimental and computational technologies.

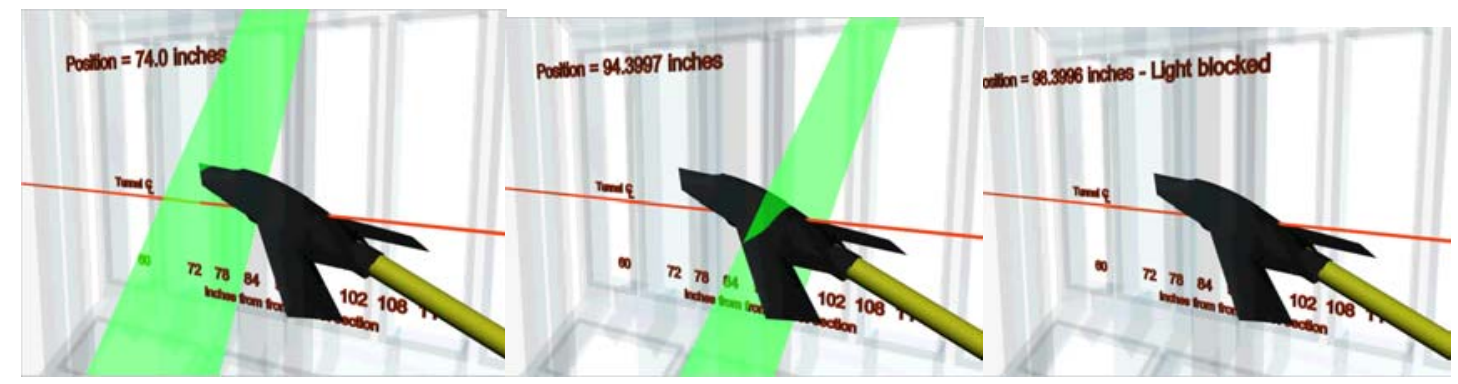

Figure 2. Lambda wing configuration model in the Subsonic Aerodynamic Research Laboratory (SARL) at Wright-Patterson Air Force Base, $\mathrm{OH}$ showing three frames from a simulation of an angleof-attack sweep that elucidated conditions in which the laser light sheet was obstructed.

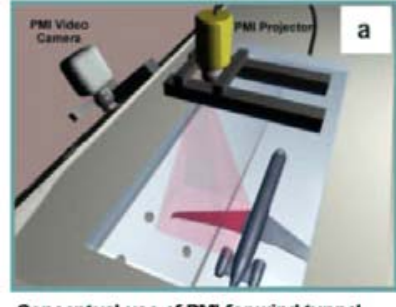

Conceptual use of PMI for wind tunnel model deformation measurements

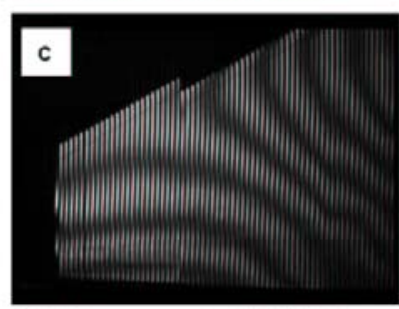

Moiré fringes generated by interfering raw PMI data with a reference grid

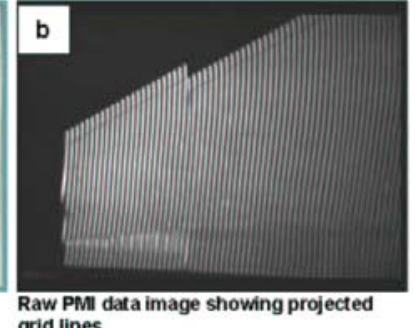
grid lines

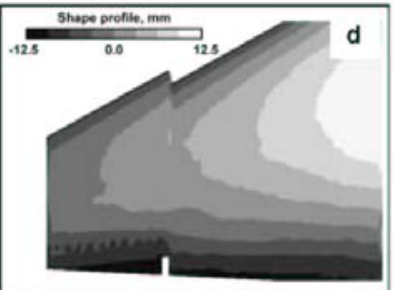

PMI-measured airfoil shape data obtained by processing Moiré fringe images

Figure 3. PMI measurement scheme for recovering the 3D geometry of test articles under aerodynamic load in aerodynamic test facilities. 

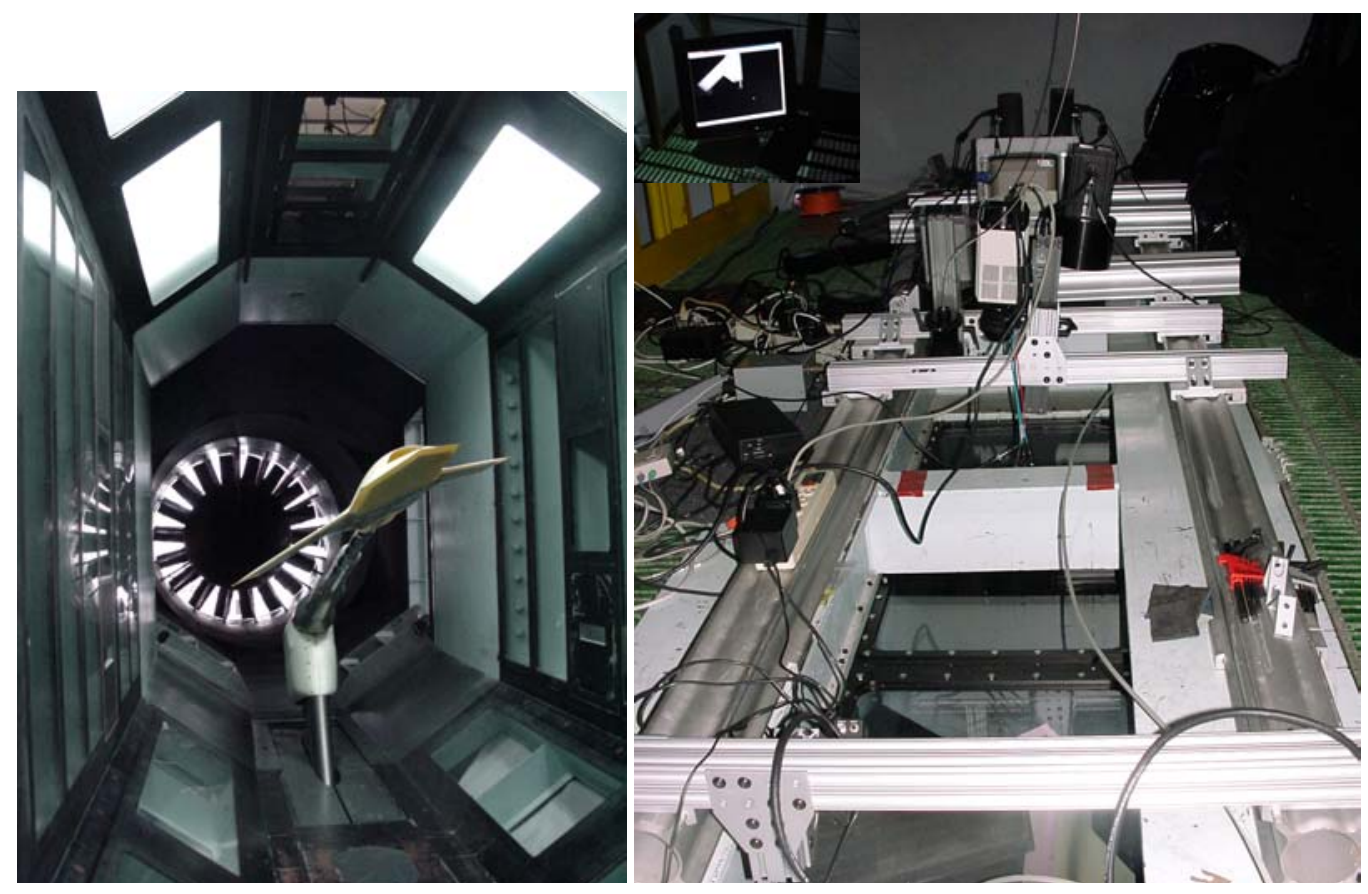

Figure 4. Lambda wind configuration model in the Subsonic Aerodynamic Research Laboratory (SARL) at Wright-Patterson Air Force Base, OH (left). Optical instrumentation mounted above test section with remote operation capability (right).

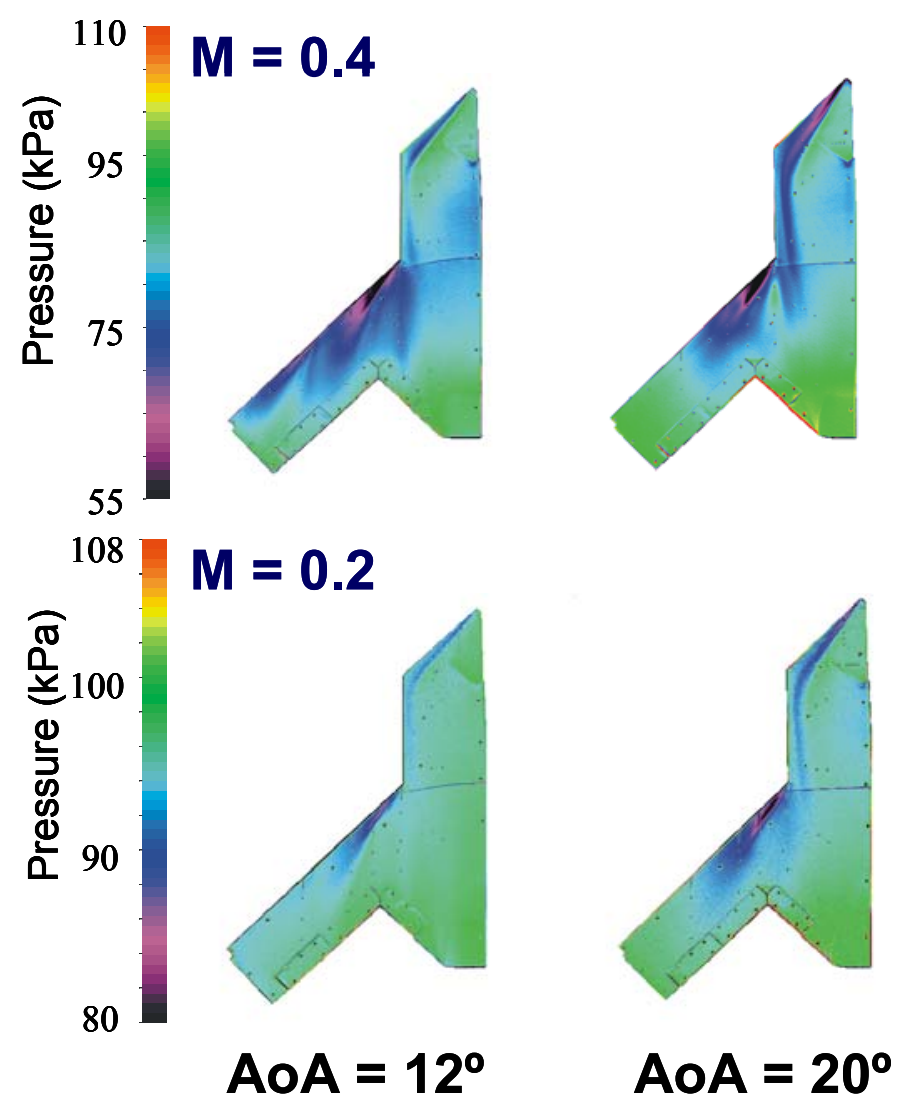

Figure 5. Intensity-based pressure-sensitive paint (PSP) results at 0.2 and 0.4 Mach number and 12 and 20 degrees angle of attack for the lambda wing base configuration. 


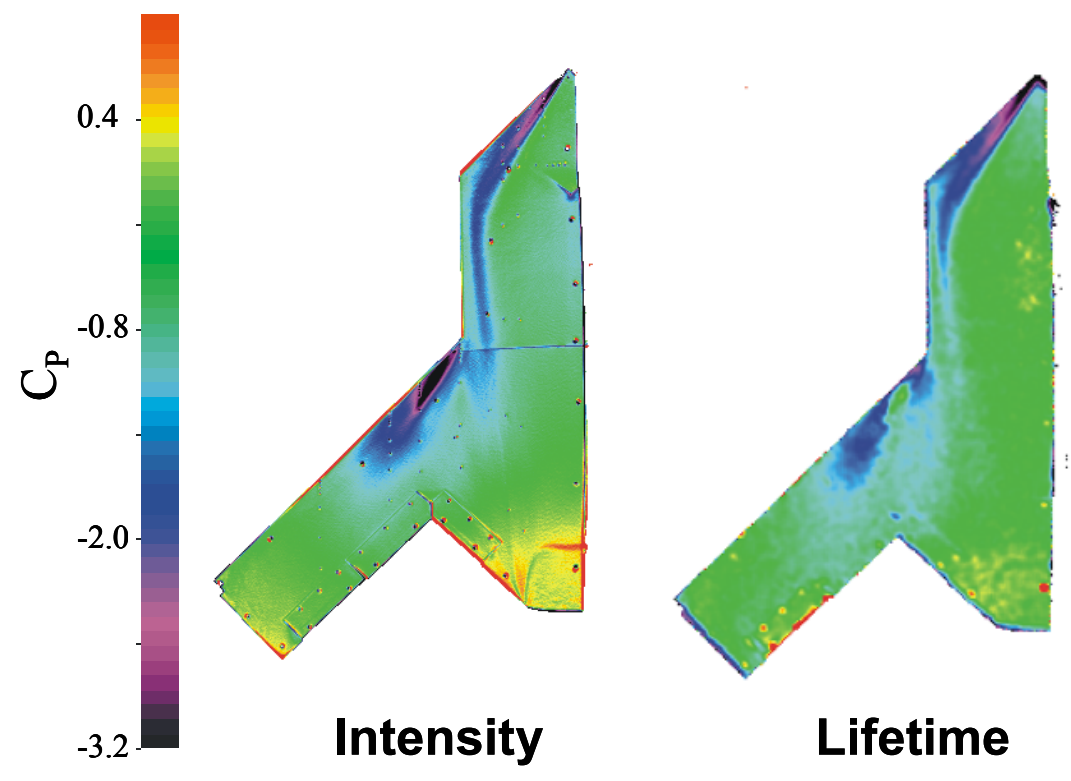

Figure 6. Comparison of intensity- and lifetime-based PSP results at 0.4 Mach number and 20 degrees angle of attack for the lambda wing base configuration. Disparity in leading edge feature resulted from tunnel debris sedimentation on model prior to lifetime image acquisition.
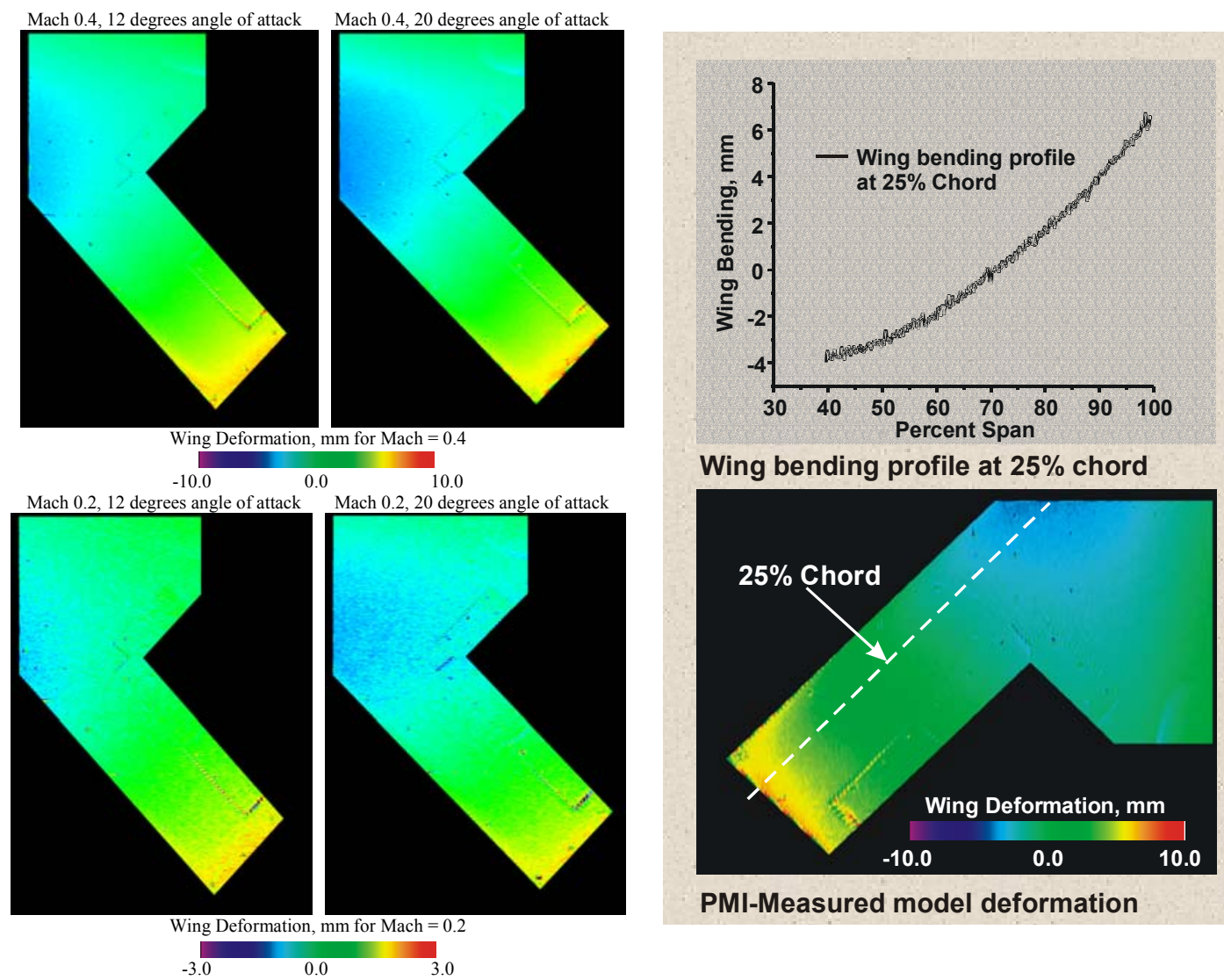

Wing bending profile at $25 \%$ chord

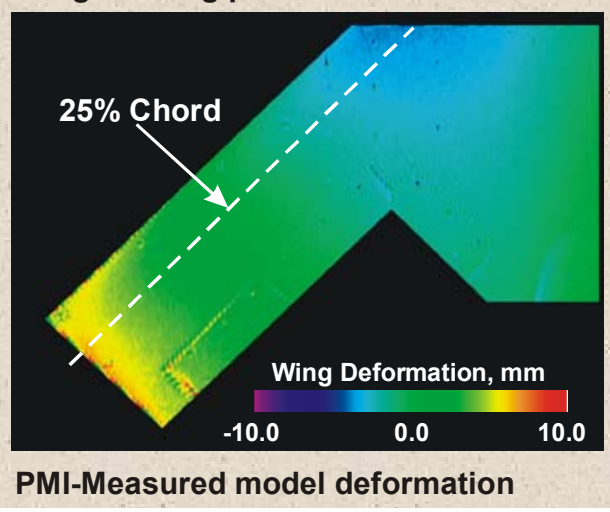

Figure 7. Projection moiré interferometry (PMI) results at 0.2 and 0.4 Mach number and 12 and 20 degrees angle of attack for the lambda wing base configuration. 
Unified PSP / PMI / ViDI in SARL
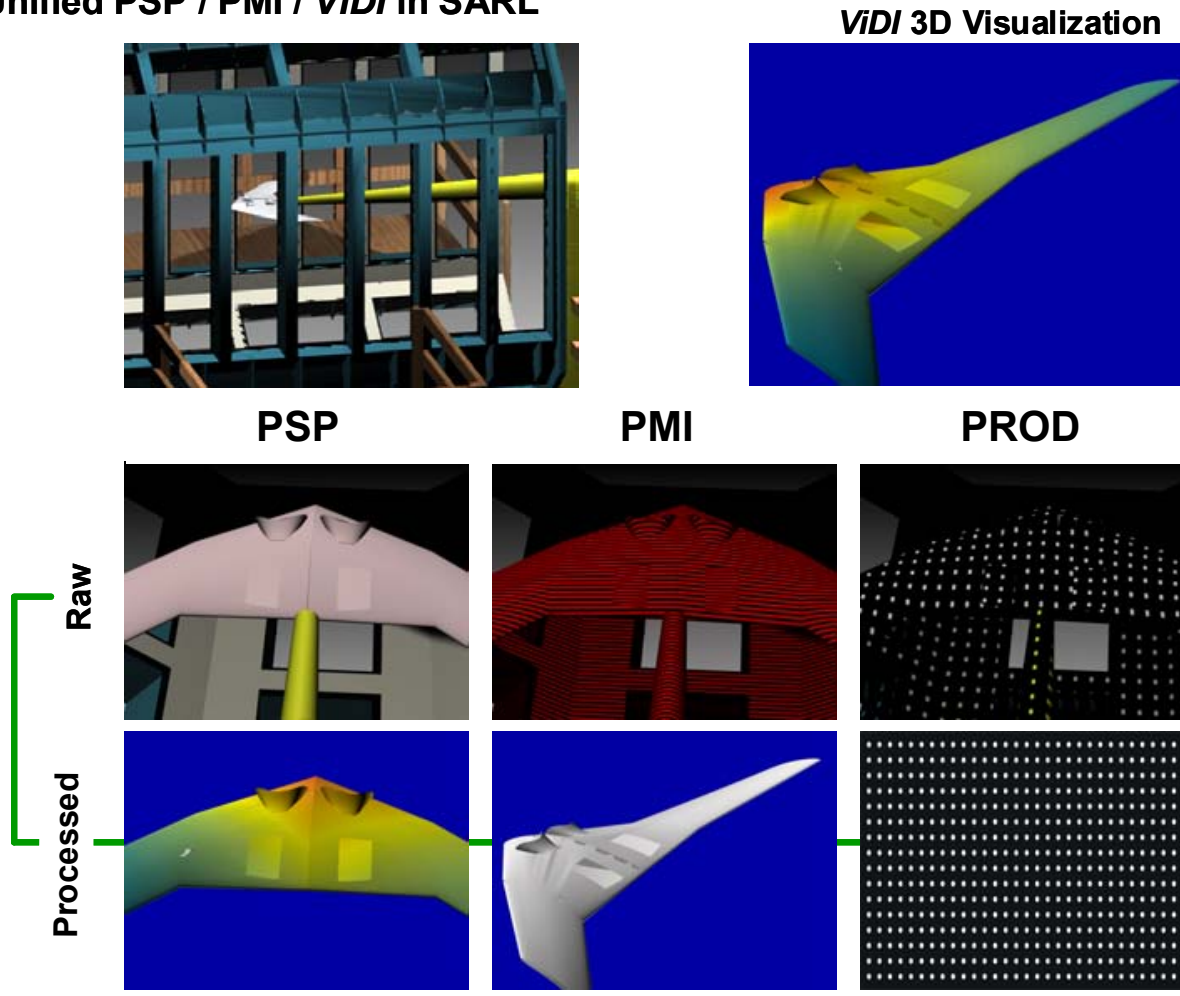

Surface Pressure

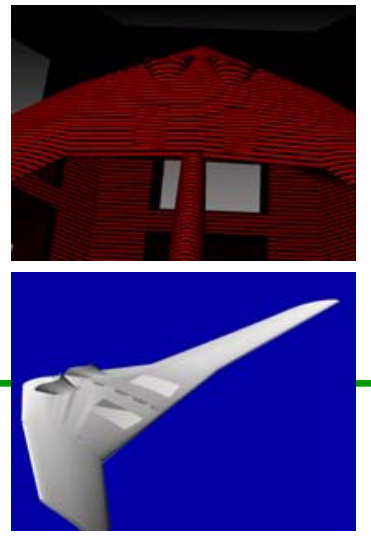

Deformation
PROD

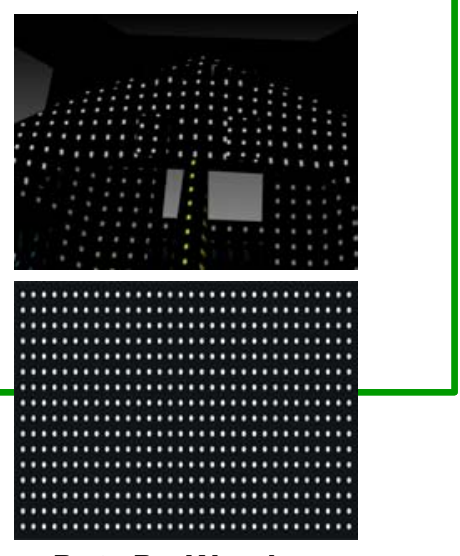

Data De-Warping

Figure 8. Schematic representation of unified lifetime PSP, PMI, PROD and ViDI for near-real-time visualization of surface pressure distributions mapped to the true model geometry.

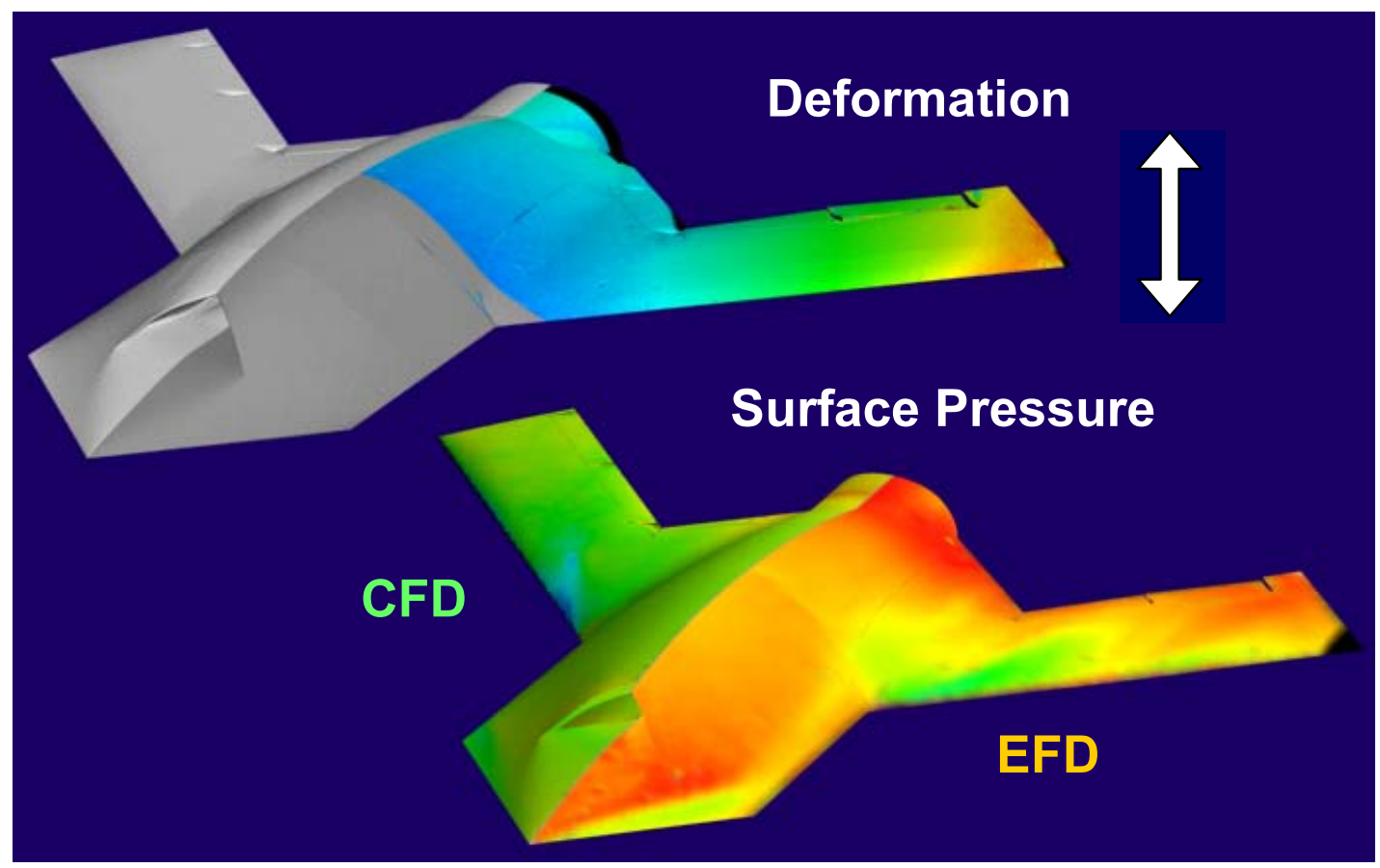

Figure 9. Application of the ViDI for the comparative evaluation of experimental (EFD) and computational fluid dynamics (CFD) results. 